\title{
THE RELATION OF SYSTOLIC UPSTROKE TIME AND PULSE PRESSURE IN AORTIC STENOSIS
}

\author{
BY \\ BENJAMIN S. BUTELER \\ From the Cardiopulmonary Laboratory, Grasslands Hospital, Valhalla, N.Y., U.S.A. \\ Received October 20, 1961
}

Entry into the left heart for the diagnosis of valvular lesions of the mitral and aortic valve is not without hazard and certainly not without varying degrees of discomfort for the patient. For these easons a continuing quest for procedures that do not require intubation of the left heart chambers persists, despite the multitude of reasonably safe and accurate approaches to the left heart itself. In 1957 Doyle and Neilson published a method of assessing the severity of aortic stenosis and for distinguishing this lesion from a coexistent but predominant aortic regurgitation. We were partizularly interested in this method because it purported to assess the severity of aortic stenosis and not merely its presence. In agreement with these authors, we had not been able to estimate even in a rough degree the magnitude of aortic stenosis from the rate of ascent of the arterial curve.

Doyle and Neilson reported their findings in 40 patients with variable degrees of aortic stenosis alone or associated with aortic regurgitation. They measured the change in systolic upstroke time against changes in pulse pressure during the first two phases of the Valsalva manœuvre in patients with sinus rhythm. The same measurement was made in beats of varying size in patients with atrial fibrillation. A regression line was plotted for each patient. In general the steeper the regression line (the longer the systolic time for larger pulse pressures or the shorter the time for smaller pressures) the more severe the stenosis. All patients with severe stenosis had steep regression lines with an absolute value of $0.10 \mathrm{sec}$. per $50 \mathrm{~mm}$. $\mathrm{Hg}$ of pulse pressure.

In order to test the validity of this method the arterial curves of 20 patients were subjected to analysis as outlined by Doyle and Neilson. Most of these patients were selected at random from patients undergoing cardiac catheterization for heart disease other than aortic stenosis or from pulmonary patients who had an arterial puncture as part of a routine pulmonary function survey. Aortic stenosis was suspected clinically in only 4 of the 20 patients. In all respects the method followed that of Doyle and Neilson with the exception that brachial artery rather than femoral artery puncture was performed. The indwelling needle was connected to a Statham strain gauge by a length of polyethylene tubing. Recordings were made on an Electronics for Medicine multipurpose recording machine and pressures were recorded at a paper speed of $75 \mathrm{~mm}$./sec. In patients with normal sinus rhythm the Valsalva manœuvre was performed by having the patient blow into a Tycos manometer to a pressure of $40 \mathrm{~mm}$. $\mathrm{Hg}$ and this pressure was sustained for 10 seconds. In all instances a significant alteration in pulse pressure was achieved. In patients with atrial fibrillation a long record was obtained sufficient to include beats of varying pulse pressures.

In 3 out of the 20 patients studied the results suggested severe aortic stenosis. In none of these patients was significant dynamic aortic stenosis present and indeed in only one of them was there any objective reason to believe that there was disease of the aortic valve.

\section{CASE RePorts}

Case 1. A 12-year-old white boy was suspected of having congenital aortic stenosis. His clinical findings consisted of a Grade IV systolic murmur over the aortic area transmitted into the neck. The aortic 
second sound was present. Radiographs of the chest were interpreted as being consistent with left ventricular enlargement with some dilatation of the ascending aorta. The electrocardiogram showed left ventricular hypertrophy. There were no ST-T wave abnormalities. A right heart catheterization demonstrated normal right-sided pressures, normal oxygen contents, and normal dye dilution curves. Fig. 1 shows the Valsalva manœuvre and Fig. 2 shows the regression line for the change in upstroke time against the change in pulse pressure. As can be seen this patient shows a change of $0.12 \mathrm{sec}$. in upstroke time for a change in pulse pressure of $10 \mathrm{~mm}$. $\mathrm{Hg}$.

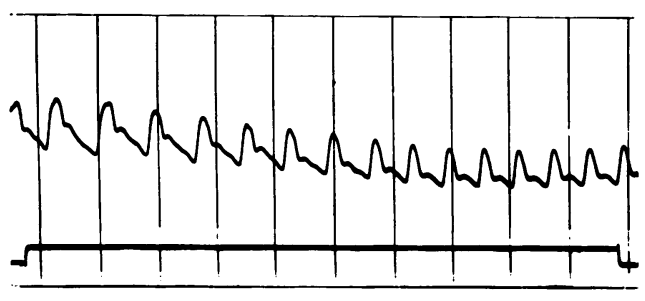

Fig. 1.-Alteration in the pulse form during the Valsalva manœuvre in Case 1.

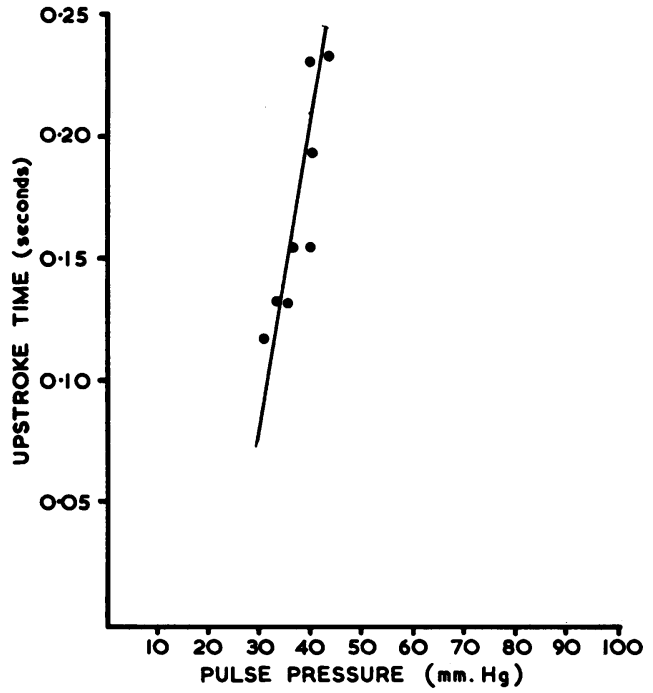

Fig. 2.-Regression line for the changes in systolic upstroke time against changes in pulse pressure during the Valsalva manœuvre in Case 1.

At operation a thrill of considerable magnitude was palpable over the base of the aorta. The aortic ring was about $2.5 \mathrm{~cm}$. in diameter and the aorta itself was dilated just distal to the ring. The left ventricle did not appear large and the wall when palpated did not appear thicker than normal. There was no subvalvular muscular hypertrophy or outflow obstruction of any kind. The aortic valve was bicuspid. The leaflets were very slightly roughened and thickened but the commissures were open to within $2 \mathrm{~mm}$. of the ring. The surgeon felt that no stenosis was present and therefore that nothing remedial could be done.

Case 2. A 35-year-old white woman presented with rheumatic heart disease, mitral stenosis, and atrial fibrillation. Physical findings consisted of a long mid-diastolic rumble, a loud mitral first sound, and an opening snap at the apex. No systolic murmurs were heard by numerous observers. Chest radiograph of the heart showed a slight cardiac enlargement with prominent pulmonary artery and enlargement of the left auricle. The cardiac catheterization data were consistent with mitral stenosis of moderate degree with rather severe myocardial failure. At no time was this patient suspected of having aortic valvular disease. Fig. 3 shows the regression line of the changes in systolic upstroke time with changes in pulse pressure due to atrial fibrillation (Fig. 4). There is a change of $0.07 \mathrm{sec}$. in systolic upstroke time for a $42 \mathrm{~mm}$. Hg change in pulse pressure. At thoracotomy the surgeon specifically looked for aortic stenosis short of opening the aorta. No thrill was present and there was nothing palpable to suggest disease of the aortic valve. A valvotomy for moderate mitral stenosis without regurgitation was performed.

Case 3. A 38-year-old white woman with rheumatic heart disease. Physical signs consisted of a middiastolic murmur at the apex with an accentuated first sound and a loud opening snap, and with atrial 


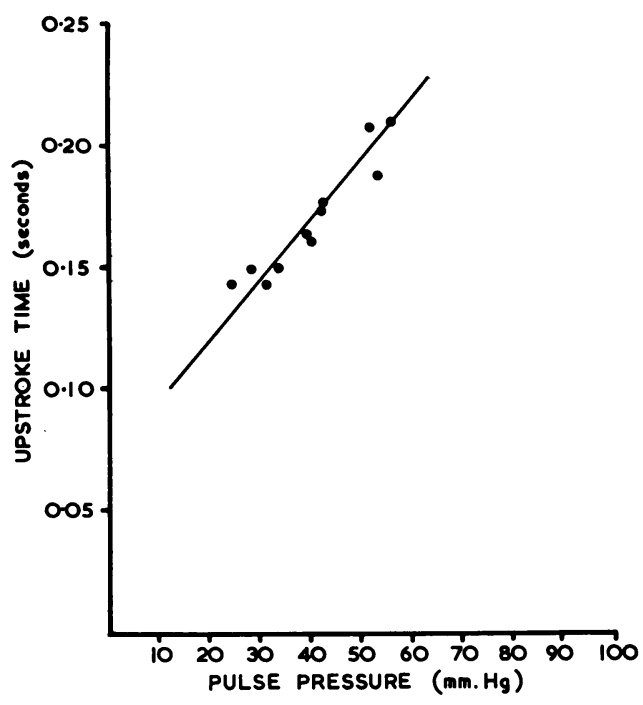

FIG. 3.-Regression line of the changes in systolic upstroke time with changes in pulse pressure due to atrial fibrillation in Case 2.

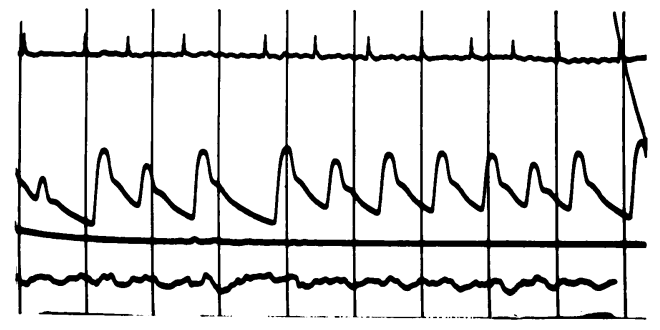

FIG. 4.-Changes in pulse form due to atrial fibrillation in Case 2. Tracing on $25 \mathrm{~mm}$. paper speed. Measurements of the systolic upstroke time were performed on a tracing at $75 \mathrm{~mm}$. speed.

fibrillation (Fig. 5). Right and left heart catheterization were consistent with the diagnosis of mitral stenosis of moderate severity. There was no gradient across the aortic valve. Fig. 6 shows the regression line of the changes in systolic upstroke time with changes in pulse pressure due to atrial fibrillation. There is a change of $0.07 \mathrm{sec} . / 42 \mathrm{~mm}$. Hg change in pressure.

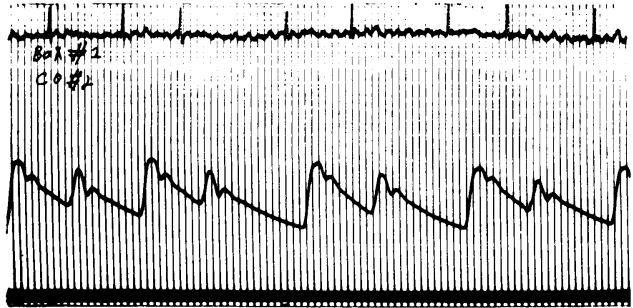

FIG. 5.-Changes in pulse form due to changes in pulse pressure due to atrial fibrillation in Case 3.

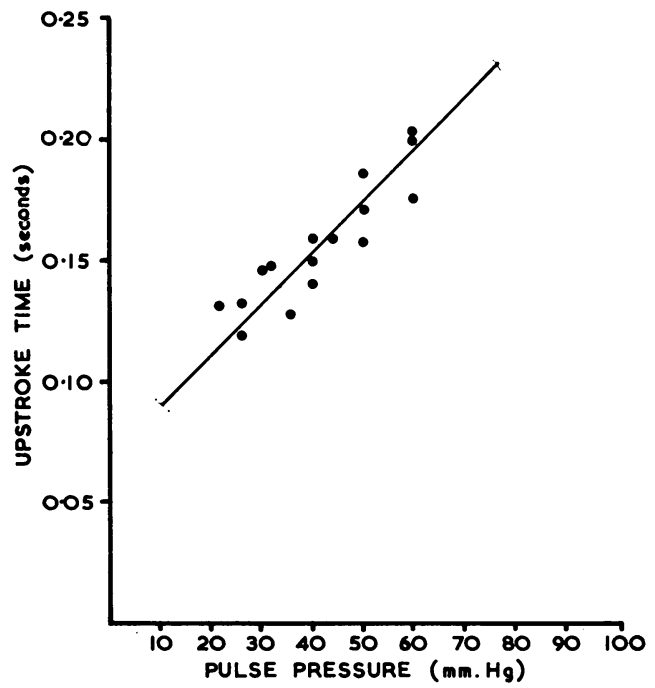

FIG. 6.-Regression line for the changes in systolic upstroke time against changes in pulse pressure due to atrial fibrillation in Case 3. 


\section{DiscussiON}

The observation that 3 patients in 20 without aortic stenosis presented changes in upstroke time consistent with the diagnosis of severe aortic stenosis according to the criteria of Doyle and Neilson casts considerable doubt upon the reliability of this method.

Goldberg, Bakst, and Bailey (1954) and Raber and Goldberg (1958) noted that in aortic stenosis the characteristic anacrotic notch disappeared during the Valsalva manœuvre. This notch was replaced by a smooth curve when the pulse pressure was small. Our Case 1 presented a conspicuous high anacrotic notch which disappeared during phase two of the Valsalva mancuvre. These changes were similar to those considered typical by Doyle and Neilson. Our patient had no demonstrably significant obstruction to the outflow tract of the left ventricle. However, the regression line as seen in Fig. 2 is consistent with severe aortic stenosis.

In our second and third case there were significant changes in systolic upstroke time in pulse pressure due to atrial fibrillation. These changes approximate the $0 \cdot 10$ change in systolic upstroke time for a change in $50 \mathrm{~mm}$. $\mathrm{Hg}$ pulse pressure considered characteristic of severe stenosis. In one of these patients there was no clinical evidence to suggest aortic stenosis and none was noted at operation. In the other there was no gradient across the aortic valve. In these two patients the changes in pulse pressure led to changes in the speed of the pulse wave. These changes were more marked in the initial rate of rise than in the total systolic time.

The major cause of the apparent positive regression lines is that in all of them there was an anacrotic shoulder or an actual bifid peak. We have noted other records in our own experience and in published reports where a small anacrotic shoulder exists. Although these records are not suitable for analysis the rate of rise appears grossly to be normal. The total systolic time might well be normal as well but because of the small shoulder systolic time during the last few millimetres of pressure the rise is longer than it might otherwise have been. It is the disappearance of this small shoulder or final peak that shortens impressively the total upstroke time. We have seen this shoulder appear and disappear during the recording of an arterial curve for no apparent reason except perhaps respiratory variation.

It seems very likely that wherever such a small late portion of the systolic upstroke occurs systolic upstroke time is bound to diminish with diminishing pulse pressure as occurs during the Valsalva manœuvre. The frequency with which an anacrotic notch occurs with aortic stenosis makes it very likely that the regression lines as obtained by Doyle and Neilson would occur with a like frequency. Unexplained is the fact that they could correlate the magnitude of this phenomenon with the magnitude of the stenosis. The fact that the regression lines remained flat (as they do in a normal subject) in aortic regurgitation may be due to the fact that in this latter disorder stroke output never falls sufficiently to cause the disappearance of the late portion of the upstroke.

\section{CONCLUSION}

In 3 patients out of 20 without aortic stenosis, and with or without atrial fibrillation, the variations in pulse pressure during the Valsalva manœuvre resemble the changes occurring in moderate or severe aortic stenosis under the same circumstances.

The findings suggest that such a relation is an unreliable guide for the detection of aortic stenosis or the assessment of its severity.

\section{REFERENCES}

Doyle, A. E., and Neilson, G. H. (1957). The Valsalva manœuvre in aortic valve disease. Brit. Heart J., 19, 525. Goldberg, H., Bakst, A. A., and Bailey, C. P. (1954). The dynamics of aortic valvular disease. Amer. Heart J., $47,527$.

Raber, G., and Goldberg, H. (1958). Left ventricular, central aortic, and peripheral pressure pulses in aortic stenosis. Amer. J. Cardiol., 1, 572. 